\section{Alteraciones del crecimiento y en el estado nutricional de pacientes pediátricos infectados con VIH}

\section{RESUMEN}

Antecedentes: la infancia es el periodo más importante de crecimiento físico y de desarrollo cognitivo de las personas. Diversos estudios muestran que el crecimiento y estado nutricional de los pacientes pediátricos infectados con VIH generalmente se encuentran alterados.

Objetivo: evaluar la prevalencia en las alteraciones en el crecimiento y en el estado nutricional de pacientes pediátricos, infectados con $\mathrm{VIH}$, atendidos en la Clínica de VIH del Instituto Nacional de Pediatría.

Materiales y métodos: estudio observacional, descriptivo, transversal, de una muestra de 49 pacientes: 22 niñas y 27 niños de entre 3 meses y 18 años de edad. Se les realizó una evaluación completa del estado nutricional en la que se consideraron indicadores antropométricos, bioquímicos, clínicos y dietéticos.

Resultados: la alteración del estado nutricional que se encontró con más frecuencia fue la desnutrición crónica compensada. Se observó una mayor prevalencia de desnutrición aguda en lactantes y preescolares. En los pacientes hospitalizados se observó una mayor frecuencia de desnutrición aguda. En la evaluación bioquímica se buscaron dislipidemias y anemia. El $46.9 \%$ de la muestra presentó alguna alteración en las concentraciones plasmáticas de triglicéridos, colesterol total, o ambos. En la evaluación clínica el síntoma que se presentó con mayor frecuencia fue la diarrea en $100 \%$ de los pacientes hospitalizados. La evaluación dietética mostró que ninguno de los pacientes cumplía con todos los criterios de una dieta correcta en su alimentación.

Conclusiones: con los resultados de este estudio se evidencia la importancia de la intervención nutricional en los pacientes de la Clínica de VIH del Instituto Nacional de Pediatría, por lo que se debe considerar incluir a un nutriólogo como parte del equipo multidisciplinario.

Palabras clave: estado nutricional, VIH, niños.

\section{Alterations of growth and nutritional status in HIV-infected pediatric patients}

\section{ABSTRACT}

Background: Childhood is the most important period of physical growth and cognitive development. Studies show that growth and nutritional status of pediatric patients infected with HIV are often altered.

\author{
Lizbeth López-Mejía ${ }^{1}$ \\ Miriam Bautista-Silva ${ }^{2}$ \\ Adriana Pinzón-Navarro² \\ Luis Xochihua-Díaz ${ }^{3}$
}

${ }^{1}$ Prácticas profesionales y realización de tesis en el Instituto Nacional de Pediatría.

2 Servicio de Nutrición del Instituto Nacional de Pediatría.

${ }^{3}$ Jefe del departamento de Infectología del Instituto Nacional de Pediatría.

Recibido: 7 de febrero 2013

Aceptado: 23 de abril 2014

Correspondencia: LN Lizbeth López Mejía Servicio de Nutrición. Instituto Nacional de Pediatría Insurgentes Sur 3700-C CP. 04530, México D.F. Teléfono: 1084-0900 ext. 1732 Celular: 044-55-32-23-48-80 lizbeth712@hotmail.com

Este artículo debe citarse como López-Mejía L, Bautista-Silva M, Pinzón-Navarro A, Xochihua-Díaz L. Alteraciones del crecimiento y en el estado nutricional de pacientes pediátricos infectados con VIH. Acta Pediat Mex 2014;35:267-279. 
Objective: To evaluate the prevalence in alterations of growth and nutritional status of HIV-infected pediatric patients treated at the HIV Clinic of the Instituto Nacional de Pediatría in México City.

Materials and Methods: It was an observational, descriptive, transversal study. The final sample included 49 patients, 22 girls and 27 boys between 3 months and 18 years old. All participants underwent a complete nutritional assessment that included anthropometric, biochemical, clinical and dietary indicators.

Results: The most common nutritional disorder was stunting. A higher prevalence of acute malnutrition was observed in infants, preschoolers and hospitalized patients. The biochemical evaluation assessed the presence of dyslipidemia and anemia. The $46.9 \%$ of the sample showed abnormal plasma levels of triglycerides, total cholesterol, or both. On clinical examination diarrhea was the most frequent symptom, which was observed in $100 \%$ of hospitalized patients. The dietary assessment showed that none of the patients followed the healthy diet baselines.

Conclusions: This study demonstrates the importance of nutritional intervention in patients with HIV. The HIV Clinic of Instituto Nacional de Pediatría should consider including a nutritionist as part of the multidisciplinary team.

Keywords: Nutritional status, HIV, Children.

\section{ANTECEDENTES}

El virus de la inmunodeficiencia humana $(\mathrm{VIH})$ pertenece a la familia de los retrovirus y a la subfamilia lentivirus. Actúa principalmente en un subgrupo de células del sistema inmunitario que poseen una molécula denominada CD4, alterando su funcionamiento y dando lugar a un estado de inmunodeficiencia. ${ }^{1,2}$

En el año 2009 la Organización Mundial de la Salud (OMS) estimó que aproximadamente 2.5 millones de niños vivían con la infección por $\mathrm{VIH}$ en el mundo. ${ }^{3}$

La infancia es el periodo más importante de crecimiento físico y de desarrollo cognitivo de las personas. Las alteraciones nutricias en esta etapa son frecuentes y mucho más hablando de niños que tienen VIH. En éstos, las alteraciones en el estado de nutrición no sólo comprometen su desarrollo sino que favorecen el avance de la enfermedad y los vuelve más vulnerables a infecciones y a otras enfermedades que deterioran su calidad de vida. ${ }^{4,5}$

La desnutrición es una importante y compleja consecuencia de la infección por VIH que se relaciona con problemas que afectan la ingestión, absorción, digestión, metabolismo y aprovechamiento de nutrimentos. ${ }^{4}$

La infección por VIH somete al paciente a un gran estrés metabólico, ocasionando un incremento en el metabolismo de proteínas que conlleva a un balance de nitrógeno negativo y a un incremento en el gasto energético. ${ }^{5}$ 
La diarrea es un problema muy común que afecta a más de $60 \%$ de los pacientes infectados, lo que genera una malabsorción crónica que termina en desnutrición. Los trastornos de la cavidad oral y esofágica por infecciones asociadas con el VIH generan pérdida de apetito e ingestión insuficiente de nutrimentos que pueden terminar en desnutrición. ${ }^{4,5}$

La pérdida de peso involuntaria, la disminución de masa corporal magra, la disminución en capacidad de fijación de hierro, la disminución del potasio sérico y la hipoalbuminemia son alteraciones frecuentes del estado nutricio de pacientes con VIH que aumentan el riesgo significativo de infecciones oportunistas y de mortalidad. ${ }^{4,6}$

La infección por VIH va acompañada de varias anormalidades nutricias específicas. Son frecuentes las deficiencias de vitaminas $A, B_{6}, B_{12}$ vitamina $\mathrm{E}$, riboflavina, cobre y zinc. ${ }^{5,7}$

El crecimiento y el estado nutricional de los pacientes pediátricos infectados con VIH con frecuencia se encuentran alterados; se ha observado que estos problemas están asociados con la baja ingestión energética, el aumento de citocinas (indicadores de inflamación) y la disminución del factor de crecimiento insulinoide (FCl-1). El déficit en el crecimiento y desarrollo se evidencia por un bajo índice de talla para la edad, circunferencia cefálica por debajo del promedio y bajo índice de peso para la talla y para la edad; esto, independientemente del tratamiento antirretroviral. ${ }^{8-11}$

Como consecuencia del uso de antirretrovirales, se han observado alteraciones en las concentraciones normales de glucosa, triglicéridos, aumento del colesterol LDL y disminución del HDL, así como aparición del síndrome de lipodistrofia, que se caracteriza por una redistribución anormal de grasa que se asocia con el desarrollo de resistencia a la insulina, diabetes mellitus, hipercolesterolemia e hipertrigliceridemia. ${ }^{12-14}$

La combinación de la infección por el VIH, que conlleva por sí misma una inmunodepresión, y el estado nutricio alterado aumentan la posibilidad de complicaciones. ${ }^{4}$

La evaluación integral del estado de nutrición en pacientes pediátricos infectados por el VIH es una herramienta útil para detectar los problemas nutricios más comunes y poder corregirlos; mejorando así el estado de los pacientes. $^{4}$

Todas las personas que se encuentran infectadas con VIH deben someterse a una evaluación nutricia completa, tanto al momento del diagnóstico como a lo largo de su enfermedad, incluso si se encuentran en la etapa asintomática. ${ }^{5}$

La evaluación del estado nutricio debe incluir cuatro parámetros básicos: antropometría, indicadores bioquímicos, indicadores clínicos e indicadores dietéticos. ${ }^{4}$

Este estudio tiene como objetivo evaluar la prevalencia en las alteraciones en el crecimiento y en el estado nutricional de pacientes pediátricos infectados con VIH, atendidos en la Clínica de VIH del Instituto Nacional de Pediatría. Conocer los principales problemas relacionados con la nutrición en esta población ayudará a crear consciencia de la importancia de considerar la participación de un nutriólogo dentro del equipo multidisciplinario de atención, para así brindar oportunamente una intervención nutricional que favorezca un desarrollo y crecimiento adecuados. Mantener un estado de nutrición óptimo en pacientes infectados con VIH es fundamental para mejorar su calidad de vida, así como para disminuir los riesgos de morbilidad y de mortalidad. ${ }^{15}$ 


\section{MATERIAL Y MÉTODOS}

Estudio observacional, descriptivo, transversal, que se llevó a cabo en el Instituto Nacional de Pediatría (INP) de la Ciudad de México, de julio a noviembre de 2012.

Como parte del manejo multidisciplinario, la Clínica de VIH del Instituto Nacional de Pediatría solicitó la intervención nutricional para sus pacientes tanto en consulta externa como en hospitalización. Para este estudio se reclutaron pacientes de ambas áreas.

Los criterios de inclusión fueron: pacientes atendidos en la Clínica de VIH del INP positivos a la prueba deVIH. Se excluyeron pacientes mayores de 18 años o aquellos cuya evaluación nutricional estuviera incompleta. Se incluyeron 51 pacientes: 22 niñas (43.1\%) y 29 niños $(56.8 \%)$ de los cuales 2 fueron excluidos por no cumplir con el rango de edad. La muestra final fue de 49 pacientes, 22 niñas (44.89\%) y 27 niños (55.1\%) de entre 3 meses y 18 años de edad. La media de edad fue de $7.69 \pm 5.76$ años.

Seis de los pacientes (12.24\%) estaban hospitalizados al momento de la evaluación nutricional, mientras que cuarenta y tres $(87.75 \%)$ fueron evaluados en la consulta externa.

Se realizó una evaluación completa del estado nutricional de los pacientes que cumplieron con los criterios de inclusión en el estudio; se recolectaron indicadores antropométricos, bioquímicos, clínicos y dietéticos.

\section{Indicadores antropométricos}

Las medidas que se tomaron fueron peso, talla o longitud, circunferencia cefálica, circunferencia media de brazo (CMB) y pliegue cutáneo tricipital (PCT). Con estas medidas se calcularon los indicadores de porcentaje de peso/talla, porcentaje de talla/edad, de peso/edad e índice de masa corporal (IMC). Los instrumentos que se utilizaron para las mediciones fueron: báscula mecánica con rango de intervalo de 50 gramos; báscula electrónica para pesar bebés con graduación de 10 gramos; estadímetro y cinta métrica con precisión milimétrica; todos marca seca y plicómetro marca Harpenden con precisión de 0.2 milímetros. Las mediciones las realizó por duplicado un investigador capacitado. En todos los casos las diferencias entre ambas mediciones fueron menores a $0.1 \mathrm{~kg}$ en el peso corporal; $0.5 \mathrm{~cm}$ en la estatura; $0.2 \mathrm{~cm}$ en circunferencia braquial y 0.2 $\mathrm{mm}$ en pliegue cutáneo tricipital.

Para el diagnóstico nutricional se utilizaron los parámetros de referencia de desnutrición de la OMS, que recomienda el uso de los índices establecidos por Gómez y Waterlow. ${ }^{16}$

Waterlow estableció los índices de peso/talla y talla/edad:

- T/E: indica alteraciones en el crecimiento lineal, definido por Waterlow como desmedro. Cuando la desnutrición afecta la talla es porque ha estado presente un largo periodo de tiempo, por lo que se habla de una desnutrición crónica.

- $\% \mathrm{P} / \mathrm{T}$ : indica alteraciones en el peso de acuerdo con la talla actual. Nos habla de un proceso agudo de desnutrición y establece la intensidad de ésta.

Gómez estableció el índice peso/edad:

- $\quad \% \mathrm{P} / \mathrm{E}:$ indica el peso del paciente en relación con el peso de niños de la misma edad. Subestima a todos los niños que están creciendo normalmente por debajo del p 50.

Para interpretar el resultado se utilizan los valores de referencia referidos en el Cuadro 1. Al combinarlos se obtiene un diagnóstico: 
Cuadro 1. Grado de desnutrición

\begin{tabular}{lcccc}
\hline Índice (\%) & Normal & Leve & Moderada & Grave \\
$\mathrm{P} / \mathrm{T}$ & $90-110$ & $80-89$ & $70-79$ & $<70$ \\
$\mathrm{~T} / \mathrm{E}$ & $>95$ & $90-95$ & $85-89$ & $<85$ \\
$\mathrm{P} / \mathrm{E}$ & $>90$ & $75-90$ & $60-74$ & $<60$
\end{tabular}

E: edad; P: peso; T: talla.

1. Eutrófico: cuando peso/talla y talla/edad se encuentran dentro de parámetros de normalidad.

2. Desnutrición aguda: cuando peso/talla es $<$ $90 \%$ y talla/edad $>95 \%$.

3. Desnutrición crónico-compensada: cuando peso/talla $>90 \%$ y talla/edad $<95 \%$.

4. Desnutrición crónico-agudizada: cuando peso/talla es $<90 \%$ y talla/edad $<95 \%$.

Para este estudio utilizamos el indicador de peso/ talla para diagnosticar la desnutrición aguda y establecer el grado de severidad de la misma. En niños menores de un año se utilizó el indicador de peso para la edad de Gómez.

El indicador de talla/edad de Waterlow se utilizó para determinar desnutrición crónica o retardo en el crecimiento lineal. Los Centers for Disease Control and Prevention (CDC) y la Academia Americana de Pediatría (AAP) recomiendan el uso del IMC para la detección de sobrepeso y obesidad en los niños, por lo que en este estudio se utilizaron las cohortes por centiles de IMC de la OMS para menores de 5 años y las de los CDC para mayores de 5 años. Con ellas se establecieron los diagnósticos de sobrepeso u obesidad. ${ }^{17}$ Para su interpretación los resultados deben ajustarse por centil según el sexo y la edad. Los CDC establecen los valores para el diagnóstico contenidos en el Cuadro 2.

Indicadores bioquímicos

Para los fines de este estudio se tomaron en cuenta lo siguientes datos: hematócrito, hemog-
Cuadro 2. Criterios para el diagnóstico de obesidad*

\begin{tabular}{lc}
\hline Diagnóstico & Centil \\
Bajo peso & $<5$ \\
Peso saludable & $5-84$ \\
Sobrepeso & $>85$ \\
Obesidad & $>95$ \\
$*$ Centers for Disease Control and Prevention
\end{tabular}

* Centers for Disease Control and Prevention

lobina, triglicéridos y colesterol total (tomados del expediente ya que se trata de análisis de rutina en el INP en los que se pide a los pacientes que se presenten con 12 horas de ayuno) de las fechas más recientes. Se utilizaron los valores de referencia de la American Heart Association para establecer parámetros de normalidad de triglicéridos y colesterol para la edad; y los de la Organización Mundial de la Salud (OMS) para la edad y así establecer parámetros de normalidad de hematocrito y hemoglobina. ${ }^{18}$ (Cuadros 3, 4 y 5)

\section{Indicadores clínicos}

Evalúa los signos y síntomas del paciente que pudieran estar relacionados con los procesos fisiológicos de digestión, absorción, utilización y excreción de nutrimentos que pudieran afectar el estado de la nutrición del niño. ${ }^{19}$

Los datos se recaudaron por medio de la exploración física y de una entrevista con el niño y padre o tutor legal.

\section{Indicadores dietéticos}

Para evaluar la dieta del paciente se utilizaron 2 herramientas: recordatorio de 24 horas, que se refiere a la recopilación de datos sobre el consumo de alimentos y bebidas del día anterior, y dieta habitual que se define como una descripción de los alimentos y bebidas más comunes que el paciente suele consumir en cada tiempo de comida. ${ }^{17}$ 
Cuadro 3. Límite inferior para establecer un diagnóstico de anemia (OMS)

\begin{tabular}{lcc}
\hline Edad & $\begin{array}{c}\text { Hemoglobina } \\
(\mathbf{g} / \mathbf{d L})\end{array}$ & $\begin{array}{c}\text { Hematócrito } \\
\mathbf{( \% )}\end{array}$ \\
\hline Recién nacidos & 16.5 & 51 \\
1 semana & 17.5 & 54 \\
2 semanas & 16.5 & 51 \\
2 meses & 11.5 & 35 \\
6 a 59 meses & 11 & 37 \\
5 a 11 años & 11.5 & 39 \\
12 a 14 años & 12 & 40 \\
Mujeres mayores de 15 años & 12 & 41 \\
Hombres mayores de 15 & 13 & 43 \\
años & & \\
\end{tabular}

Cuadro 4. Concentraciones normales de triglicéridos (mg/dL) según edad

\begin{tabular}{lcc}
\hline Edad & Hombres & Mujeres \\
\hline 0-7 días & $21-182$ & $28-166$ \\
8 a 30 días & $30-184$ & $30-165$ \\
31-90 días & $40-175$ & $35-282$ \\
91-180 días & $45-291$ & $50-355$ \\
181-365 días & $45-501$ & $35-431$ \\
1-3 años & $27-125$ & $27-125$ \\
4-6 años & $32-116$ & $32-116$ \\
7-9 años & $28-129$ & $28-129$ \\
10-11 años & $24-137$ & $39-140$ \\
12-13 años & $24-145$ & $37-130$ \\
14-15 años & $34-165$ & $38-135$ \\
16-19 años & $34-140$ & $37-140$ \\
\hline
\end{tabular}

Cuadro 5. Concentraciones normales de colesterol total (mg/dL) según edad

\begin{tabular}{lcc}
\hline Edad & Hombres & Mujeres \\
1-1 mes & $54-151$ & $62-155$ \\
2-6 meses & $81-147$ & $62-141$ \\
7-12 meses & $76-179$ & $76-216$ \\
1-3 años & $85-192$ & $108-193$ \\
4-6 años & $110-217$ & $106-193$ \\
7-9 años & $110-211$ & $104-210$ \\
10-12 años & $105-223$ & $105-218$ \\
13-15 años & $91-204$ & $108-205$ \\
16-18 años & $82-192$ & $92-234$
\end{tabular}

Para fines de este estudio se definió como dieta correcta aquella que cumple con las siguientes características: ${ }^{17}$

- Completa: que contenga todos los nutrimentos. Incluir en cada tiempo principal de comida un alimento de cada grupo (frutas y verduras, cereales y tubérculos, leguminosas $y$ alimentos de origen animal).

- Equilibrada: que los nutrimentos guarden las proporciones adecuadas entre sí.

- Inocua: que su consumo habitual no implique riesgos para la salud.

- Suficiente: que cubra las necesidades de todos los nutrimentos.

- Variada: que incluya diferentes alimentos de cada grupo en las comidas.

- Adecuada: acorde con los gustos y con la cultura.

La evaluación la realizó la nutrióloga encargada del proyecto. En los casos necesarios se pidió el apoyo de otro nutriólogo para la toma de las medidas antropométricas y con el fin de disminuir el rango de error.

A todos los participantes se les otorgó orientación nutricional: un tríptico para prevención de dislipidemias, un plan de alimentación individualizado y se les dio seguimiento nutricional a aquellos que lo requerían como beneficio secundario a su participación en el estudio.

\section{Análisis estadístico}

Se realizaron pruebas estadísticas descriptivas, cuantitativas y cualitativas. Para las variables numéricas se utilizaron promedios y desviaciones estándar, para las variables categóricas se calcularon las prevalencias de las alteraciones nutricias a través de proporciones. Se utilizó la prueba de Pearson para realizar correlaciones entre dos variables cuantitativas continuas. 
López-Mejía L et al. Alteraciones nutricionales y del crecimiento en pacientes con VIH

\section{RESULTADOS}

Las medias y desviaciones estándar de los datos recaudados para la evaluación nutricional se muestran en el Cuadro 6.

Los resultados de la evaluación nutricional mostraron lo siguiente:

La evaluación antropométrica mostró que de la totalidad de pacientes incluidos en el estudio $12(24.48 \%)$ se encontraban eutróficos; es decir, todos sus parámetros antropométricos se encontraron dentro de los rangos normales. 4 pacientes $(8.16 \%)$ presentaron obesidad, 5 (10.2\%) sobrepeso; 4 (8.16\%) desnutrición crónica agudizada de intensidad leve; 3 pacientes $(6.12 \%)$ desnutrición crónica agudizada de intensidad moderada; $2(4.08 \%)$ desnutrición crónica agudizada de intensidad severa y 24 (48. 9\%) desnutrición crónica de acuerdo con el indicador T/E descrito por Waterlow. ${ }^{16}$ (Cuadro 7)

La muestra se estratificó por género. (Figura 1)

Cuadro 6. Medias y desviaciones estándar de la evaluación nutricional

\begin{tabular}{|c|c|c|}
\hline & Femenino & Masculino \\
\hline $\begin{array}{l}\text { Total de pacientes } \\
\text { evaluados }\end{array}$ & 22 & 27 \\
\hline Edad (años) & $8.1( \pm 6.12)$ & $6.04( \pm 5.3)$ \\
\hline Peso (kg) & $26.5( \pm 16.56)$ & $27( \pm 16.76)$ \\
\hline Talla (cm) & $117.3( \pm 32.25)$ & $110.6( \pm 35.63)$ \\
\hline$\% \mathrm{P} / \mathrm{T}$ & $102.08( \pm 12.39)$ & $94.21( \pm 12.37)$ \\
\hline$\% \mathrm{~T} / \mathrm{E}$ & $91.4( \pm 4.22)$ & $93.14( \pm 5.18)$ \\
\hline \multirow[t]{2}{*}{$\begin{array}{l}\% \mathrm{P} / \mathrm{E} \text { (menores de } \\
1 \text { año) }\end{array}$} & 75.88(-) & $56.63( \pm 7.45)$ \\
\hline & $17.04( \pm 3.95)$ & $15.75( \pm 2.51)$ \\
\hline IMC $\left(\mathrm{kg} / \mathrm{m}^{2}\right)$ & centil $50( \pm 30)$ & centil $35.9( \pm 27.8)$ \\
\hline $\begin{array}{l}\text { Triglicéridos } \\
\text { (mg/dL) }\end{array}$ & $204( \pm 209)$ & $134( \pm 54.5)$ \\
\hline $\begin{array}{l}\text { Colesterol total } \\
(\mathrm{mg} / \mathrm{dL})\end{array}$ & $182.36( \pm 57.6)$ & $160( \pm 25.97)$ \\
\hline $\begin{array}{l}\text { Hemoglobina } \\
(\mathrm{g} / \mathrm{dL})\end{array}$ & $13.45( \pm 1.34)$ & $13.2( \pm 1.52)$ \\
\hline Hematócrito (\%) & $39.21( \pm 3.84)$ & $38.42( \pm 4)$ \\
\hline
\end{tabular}

Cuadro 7. Diagnóstico nutricional de acuerdo con la evaluación antropométrica

\begin{tabular}{lcc}
\hline Diagnóstico nutricional & $\begin{array}{c}\text { No. de } \\
\text { pacientes }\end{array}$ & $\%$ \\
& 12 & 24.48 \\
Eutrófico & 4 & 8.16 \\
Obesidad & 5 & 10.20 \\
Sobrepeso & 4 & 8.16 \\
Desnutrición crónica agudizada leve & 3 & 6.12 \\
Desnutrición crónica agudizada & & \\
moderada & 2 & 4.08 \\
Desnutrición crónica agudizada severa & 24 & 48.90 \\
Desnutrición crónica & 49 & - \\
Total de pacientes evaluados & &
\end{tabular}

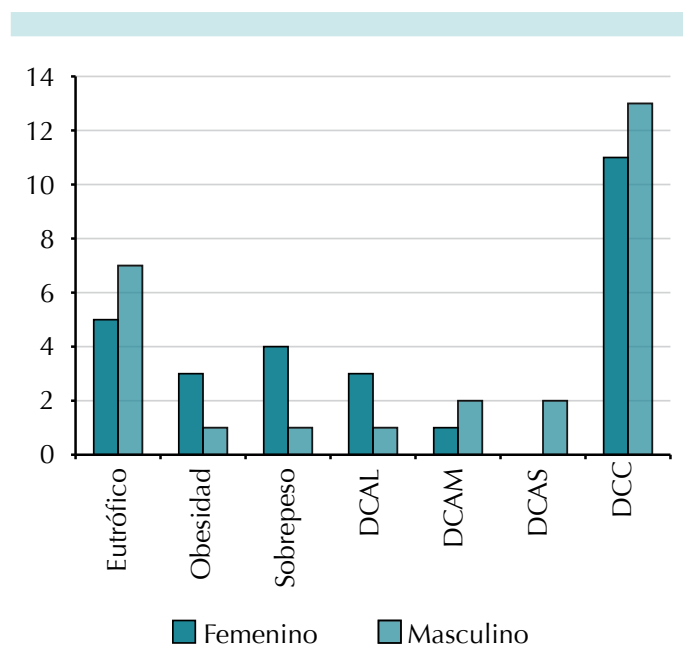

DCAL: Desnutrición crónica agudizada leve; DCAM: Desnutrición crónica agudizada moderada; DCAS: Desnutrición crónica agudizada severa; DCC Desnutrición crónica compensada.

Figura 1. Diagnóstico nutricional según género.

La desnutrición aguda se presentó en un total de 9 pacientes. Se observó una mayor prevalencia de desnutrición aguda en lactantes y preescolares.

Al comparar la desnutrición aguda en pacientes hospitalizados en comparación con pacientes ambulatorios se observó que $66.6 \%$, es decir 4 de cada 6 pacientes hospitalizados, presentó 
algún grado de desnutrición aguda. En los pacientes ambulatorios la desnutrición aguda se presentó en 5 de 43 pacientes (11.6\%). (Figura 2)

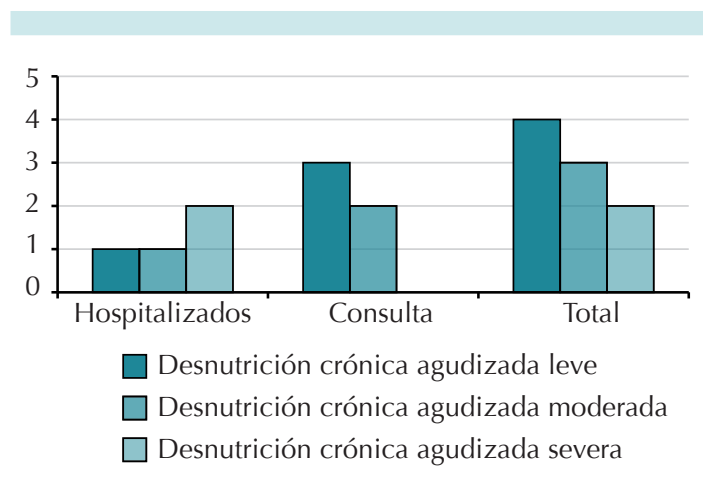

Figura 2. Desnutrición aguda en pacientes hospitalizados y ambulatorios.

Como parte integral del tratamiento a los pacientes hospitalizados se les dio seguimiento y soporte nutricional durante su estancia. En todos ellos se observó una recuperación nutricional al alza.

Para la evaluación antropométrica se tomaron medidas del perímetro braquial y del pliegue cutáneo tricipital para estimar las masas grasa y magra de los pacientes. Los resultados se muestran en las Figuras 3 y 4.

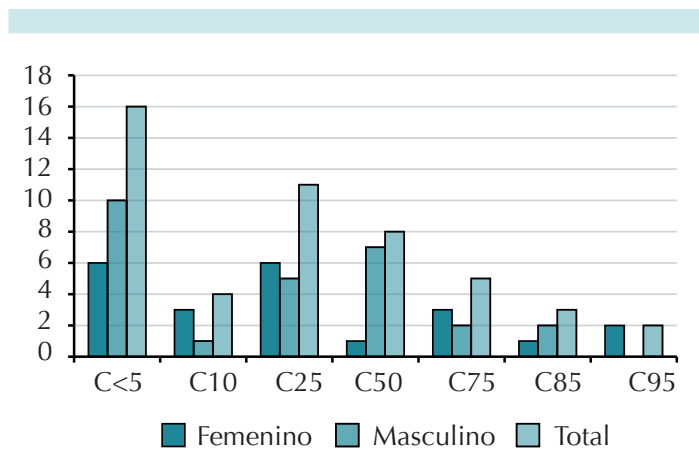

Figura 3. Evaluación del perímetro braquial (PB).

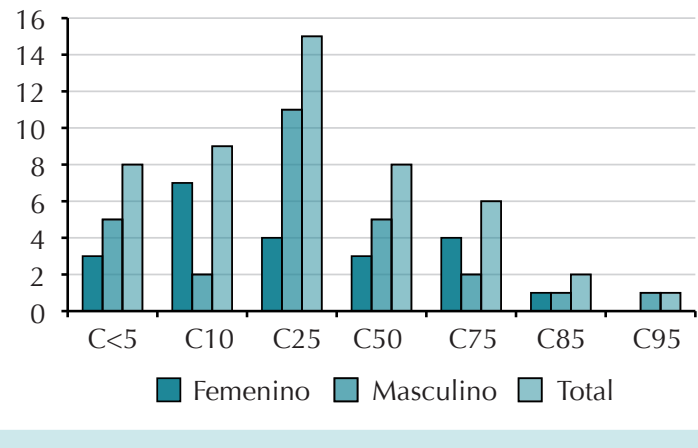

Figura 4. Evaluación del pliegue cutáneo tricipital (PCT).

Se evaluó la circunferencia cefálica de los pacientes menores a 36 meses: de 12 niños valorados 5 tuvieron un circunferencia cefálica menor al centil 5, lo cual representa el $41.6 \%$. En todos los pacientes se utilizó la edad cronológica ajustada a la talla para esta evaluación con el fin de no subestimar la medición.

En cuanto a la evaluación bioquímica se buscaron dislipidemias y anemia.

De la muestra 23 pacientes (46.9\%) presentó alguna alteración en los niveles plasmáticos de triglicéridos, colesterol total, o ambos. Por tratarse de un estudio transversal sólo se tomó una muestra; es importante tomar por lo menos otra muestra para confirmar el diagnóstico. En la Figura 5 se muestran las dislipidemias y se comparan por género. El $45.45 \%$ de las niñas (10) presentó hipertrigliceridemia y el $41 \%$ (9) hipercolesterolemia. En los niños se observó hipertrigliceridemia en $40.74 \%$ (11) e hipercolesterolemia en $7.4 \%$ (2).

Utilizando la prueba de Pearson se buscaron correlaciones entre el tiempo con tratamiento antirretroviral y las concentraciones de triglicéridos y de colesterol total. Ambas correlaciones fueron estadísticamente significativas. El coeficiente de correlación (R) entre 


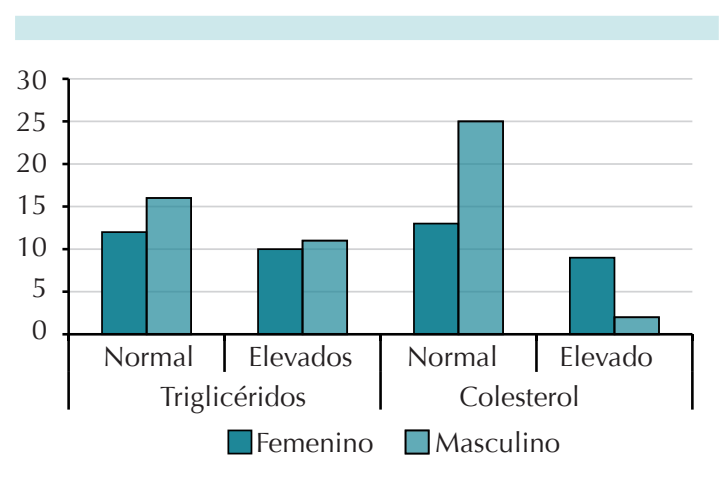

Figura 5. Frecuencia de dislipidemias según género.

las concentraciones de triglicéridos y el tiempo con tratamiento antirretroviral fue de 0.43 y entre las concentraciones de colesterol total y el tiempo con tratamiento antirretroviral fue de 0.19. (Figuras 6 y 7 )

Según los criterios de la OMS para el diagnóstico de anemia ésta se presentó en $8.16 \%$ (4 pacientes) de la muestra total. Todos los pacientes que la presentaron se encontraban hospitalizados.

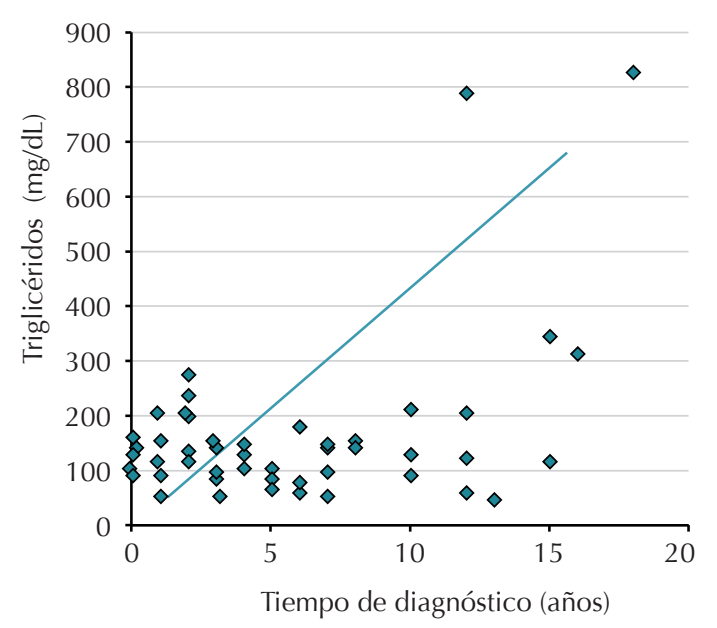

Figura 6. Correlación entre triglicéridos y tiempo con tratamiento antirretroviral.

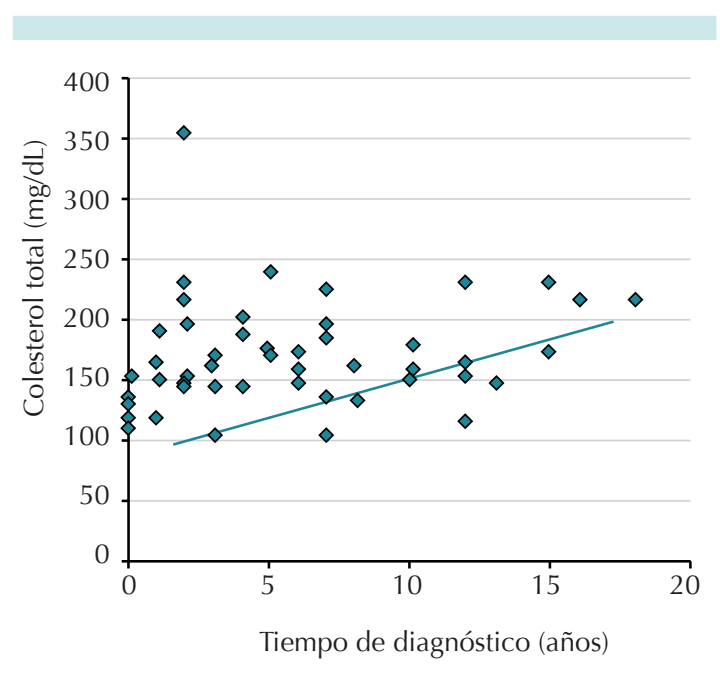

Figura 7. Correlación entre colesterol total y tiempo con tratamiento antirretroviral.

En la evaluación clínica el síntoma gastrointestinal que se observó con mayor frecuencia fue la diarrea; la tuvieron 9 pacientes (18.3\%): 6 de los 6 pacientes hospitalizados (100\%) pero sólo $3(6.97 \%)$ de los pacientes ambulatorios.

Otras manifestaciones clínicas entre los pacientes hospitalizados fueron: náuseas (1 paciente), distensión abdominal (2 pacientes) y problemas orales (1 paciente). Los pacientes ambulatorios no presentaron otras alteraciones clínicas que pudieran afectar su estado nutricional. (Figura 8)

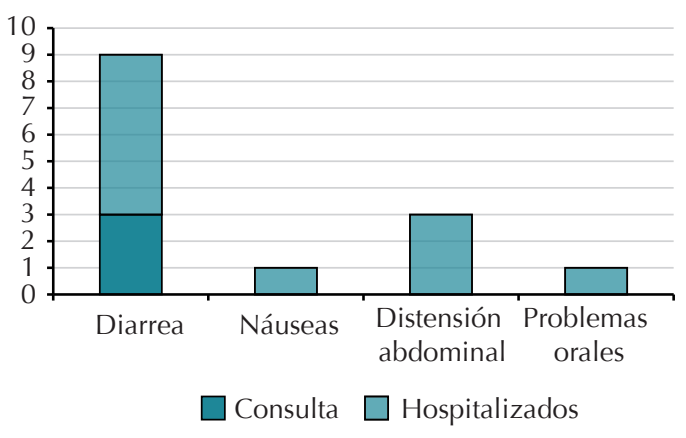

Figura 8. Síntomas clínicos. 
La evaluación dietética mostró que ningún paciente cumplía con todos los criterios de la dieta correcta en su alimentación. La dieta de 37 de los pacientes $(75.5 \%)$ era incompleta; la de 24 (48.9\%) no fue variada; la de 38 (77.55\%) fue desequilibrada; la de 33 (67.34\%) inadecuada (ya que era alta en azúcares simples y grasas saturadas) y, finalmente, la dieta de 16 de los niños evaluados era insuficiente (32\%). (Figura 9)

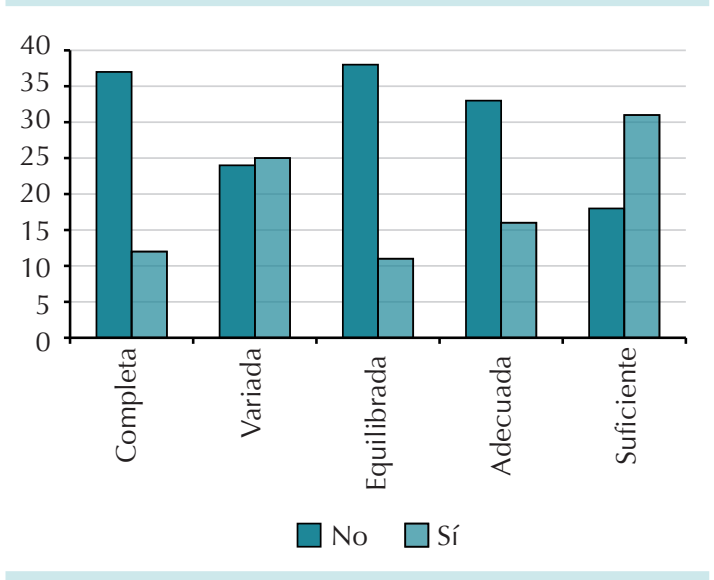

Figura 9. Evaluación de la dieta correcta.

\section{DISCUSIÓN}

De acuerdo con los resultados del estudio existió alta prevalencia de alteraciones en el estado nutricional de los pacientes pediátricos infectados con $\mathrm{VIH}$.

En este estudio encontramos 24 pacientes con talla baja para la edad, pero con un peso adecuado; esto, según la clasificación de Waterlow, nos indica una desnutrición crónica compensada. Sin embargo, la talla baja puede deberse a múltiples factores, incluyendo los genéticos. No se contó con los datos para la obtención de la talla blanco familiar por lo que es imposible afirmar que la talla baja se debió a factores nutricionales. Sin embargo, se sabe que múltiples factores pueden estar relacionados con el crecimiento deficiente en niños infectados con $\mathrm{VIH}$.
Por ejemplo, dieta inadecuada, complicaciones de salud, retardo en el crecimiento intrauterino y el diagnóstico por sí mismo. ${ }^{20}$

Un estudio de revisión realizado en la Universidad de Columbia, Nueva York, por Arpadi S, reportó que $50 \%$ de los niños infectados con VIH presentaba alteraciones en el crecimiento lineal; así mismo, en un estudio realizado por Villalobos et al se observó una alteración en el indicador de talla para la edad en $56.7 \%$ de los niños evaluados. Estos resultados concuerdan con el $48.9 \%$ de niños con desnutrición crónica compensada de nuestro estudio. , $^{9,21}$

Con respecto a la dieta podemos señalar que al recordatorio de 24 horas ninguno de los pacientes evaluados cumplió con todos los criterios de la dieta correcta (completa, variada, equilibrada, adecuada, suficiente e inocua) por razones multifactoriales dependientes de educación, nivel económico, gustos y creencias culturales. Esto puede influir en el crecimiento de los niños ya que pueden existir deficiencias tanto de macrocomo de micronutrimentos. Un aporte deficiente de energía se observó en 32\% de los pacientes, lo cual puede contribuir a la desnutrición y a otras deficiencias nutricionales.

Los pacientes con VIH pueden presentar complicaciones gastrointestinales e infecciosas frecuentes, especialmente antes del diagnóstico, que afectan su estado nutricional por catabolismo o disminución de absorción de nutrimentos cuando se presentan problemas como diarrea, observada en todos los pacientes hospitalizados en nuestro estudio. En un análisis realizado por Villalobos y sus colaboradores $46.7 \%$ de los niños presentó diarrea; sin embargo, no se reportó si se trataba de pacientes hospitalizados o ambulatorios. ${ }^{9}$

Estas condiciones conllevan a la presencia de desnutrición aguda. Los niños, por encontrarse en una etapa de crecimiento y maduración de 
órganos y sistemas, son especialmente vulnerables a padecer desnutrición; en particular los lactantes y los niños en edad preescolar pues sus reservas son menores a las de los niños más grandes. ${ }^{4}$ Situación que se ejemplifica en este estudio ya que 6 de los 9 pacientes que presentaron desnutrición aguda tenían menos de 3 años de edad.

La desnutrición hospitalaria es un problema frecuente. Se estima que la prevalencia de desnutrición hospitalaria infantil varía de 20 a 65\% entre los niños atendidos en hospitales de Estados Unidos, Inglaterra, Europa y América Latina. Los niños que se encuentran inmunodeprimidos tienen mayor riesgo de padecer desnutrición. Los pacientes pediátricos infectados con VIH pasan largos periodos de tiempo hospitalizados antes de establecer su diagnóstico, mientras son controlados y cuando presentan complicaciones posteriores. Esta situación los pone en riesgo de padecer desnutrición aguda. ${ }^{22}$

En la evaluación de los pacientes hospitalizados de este estudio se observó desnutrición crónica agudizada, con diferentes grados de severidad, en $66.6 \%$ de los pacientes.

La anemia se evaluó según los criterios de la OMS y se observó en 4 de los 6 pacientes hospitalizados. La anemia puede presentarse por múltiples causas, por ejemplo puede ser el resultado de trastornos hereditarios, deficiencias nutricionales (hierro, vitamina B12 y ácido fólico), por ingestión insuficiente o malabsorción, infecciones o por la exposición a fármacos. 6,18,23 Todos los niños del estudio que presentaron anemia durante su evaluación nutricional estaban hospitalizados y presentaban problemas de diarrea y desnutrición aguda. En este caso no se pudo establecer la causa específica de la anemia a través de estudios bioquímicos, por falta de los datos del volumen corpuscular medio (VCM) y de hemoglobina corpuscular media (HCM).
Otros problemas nutricionales como el sobrepeso y la obesidad también se encontraron en la población estudiada, aunque con menores prevalencias. La presencia de estos problemas nutricionales puede deberse a estilos de vida poco saludables que incluyen malos hábitos de alimentación y sedentarismo. Como ya se mencionó, ningún paciente cumplió con todos los criterios de una dieta correcta. En los pacientes con sobrepeso y obesidad se observó ingesta alta de grasas saturadas y consumo excesivo de energía en su recordatorio de 24 horas; lo cual puede asociarse con sobrepeso y obesidad.

Las prevalencias de hipercolesterolemia y de hipertrigliceridemia en los niños infectados con VIH, atendidos en el Instituto Nacional de Pediatría (México), fueron similares a las descritas por otros autores. Se encontró que $46.9 \%$ del total de pacientes evaluados tenía alguna alteración en las concentraciones plasmáticas de triglicéridos, colesterol total, o ambos. El $43.09 \%$ de los pacientes tuvo hipertrigliceridemia, mientras que $24.2 \%$ presentó hipercolesterolemia. Del $46.9 \%$ de los pacientes con alguna alteración lipídica 38.9\% no mostraba obesidad ni sobrepeso y su dieta no era rica en azúcares simples ni en grasas saturadas.

Existió una correlación $(\mathrm{R}=0.43, p<0.001)$ entre el tiempo con tratamiento antirretroviral y las concentraciones de triglicéridos en la sangre. Es importante mencionar que todos los niños incluidos en este estudio se encontraban con tratamiento antirretroviral.

Aunque se puede pensar que las dislipidemias pueden relacionarse con efectos secundarios de los antirretrovirales en este estudio no se pudo establecer la causa exacta de las dislipidemias porque, al tratarse de un estudio transversal, se realizaron mediciones únicas. ${ }^{24}$

Es necesario efectuar un estudio longitudinal para correlacionar los tipos de antirretrovirales, 
dosis y tiempo de exposición, con la presencia de dislipidemias en pacientes pediátricos infectados por VIH.

La terapia antirretroviral ha modificado el curso evolutivo del VIH transformando a esta enfermedad en un padecimiento crónico controlable. ${ }^{9}$ Sin embargo, tras aumentar la supervivencia de los pacientes y por el tiempo de administración de los medicamentos antirretrovirales, se han observado efectos adversos como las dislipidemias que pueden implicar factores de riesgo cardiovascular en pacientes con $\mathrm{VIH} .{ }^{25,26}$ Existen varios estudios que respaldan lo descrito previamente. Por ejemplo, en un estudio realizado en Uruguay por Gutiérrez y sus colegas, que evaluó la prevalencia de dislipidemias en niños $\mathrm{VIH}$ positivos con tratamiento antirretroviral, encontraron que $55 \%$ tuvo alguna alteración en las concentraciones totales de colesterol, triglicéridos, o ambos. ${ }^{27}$ Otro estudio realizado en Cali, Colombia, por López Pío et al y que también evaluó dislipidemias en niños que recibían un esquema de tres antirretrovirales, se observó que $44.4 \%$ de los niños presentaron hipertrigliceridemia y $22.22 \%$ hipercolesterolemia. ${ }^{28}$

En el estudio de Villalobos y sus colaboradores el colesterol sérico, en promedio, se encontró dentro de los rangos normales para los grupos etarios; sin embargo, se observaron valores de triglicéridos superiores a los cortes de referencia establecidos para Venezuela. ${ }^{9}$

A largo plazo, las dislipidemias y la obesidad ocasionan problemas cardiovasculares que pueden poner en riesgo la vida del paciente. Es importante otorgar a estos pacientes tratamiento nutricional..$^{29,30}$

En Latinoamérica no existen suficientes estudios que muestren las alteraciones en el estado nutricional de niños infectados con VIH, por lo que es necesaria más investigación.
Los resultados obtenidos en este estudio evidencian la importancia de la intervención nutricional en los pacientes pediátricos infectados por $\mathrm{VIH}$, tanto al momento del diagnóstico como a lo largo de su vida. Cada periodo de la enfermedad conlleva un riesgo nutricional y más aún por tratarse de población pediátrica. Por ejemplo, los pacientes de diagnóstico reciente tienen mayor riesgo de padecer desnutrición aguda con secuelas importantes en el desarrollo y crecimiento; sobre todo si se trata de lactantes o preescolares. ${ }^{4}$ Los pacientes que ya están controlados por medio de fármacos antirretrovirales se encuentran en riesgo de padecer alteraciones en el metabolismo lipídico. ${ }^{9,31,32}$

Por otro lado, durante el desarrollo del estudio se observó que son pocos los pacientes y sus familiares con consciencia de la importancia que tiene la correcta alimentación para ayudar a mejorar su salud. Esto se debe a la falta de información y orientación nutricional.

Se debe considerar incluir a un nutriólogo como parte del equipo multidisciplinario de la Clínica de VIH del Instituto Nacional de Pediatría (México) ya que todos sus pacientes se encuentran en riesgo de padecer alguna alteración nutricional. El tratamiento integral de estos pacientes que incluya una intervención nutricional oportuna, tanto para la prevención de alteraciones nutricionales como para el tratamiento de aquellos que ya cursan con alguna alteración, es esencial para mejorar el estado de salud de los pacientes de la Clínica de VIH y para brindarles una mejor calidad de vida. ${ }^{4}$

\section{REFERENCIAS}

1. Sociedad Andaluza de Enfermedades Infecciosas. La infección por VIH: Guía Práctica. 2o edición. España: Sociedad Andaluza de Enfermedades Infecciosas; 2003.

2. Lamotte J. Infección y enfermedad por VIH/SIDA. Medi San 2004;8(4):49-63. 
López-Mejía L et al. Alteraciones nutricionales y del crecimiento en pacientes con VIH

3. ONUSIDA. UNAIDS Report on the global AIDS epidemic. ONU;2010.

4. Kathleen L, Escott-Stump S. Krause Dietoterapia. 12 edición. España: Elsevier Masson;2009.

5. Morales AJ, Sánchez SM, Linares S, Nandí L, Villalobos A, Cashat $\mathrm{C}$, et al. Condición nutricia y apoyo alimentario en niños con VIH. Bol Med Hosp Infant Mex 2002;59:250-64.

6. Irlam JH, Siegfried N, Visser ME, Rollins NC. Micronutrient supplementation for children with HIV infection. Cochrane Database of Systematic Reviews 2013, Issue 10. Art. No.: CD010666.

7. Johann-Liang R, O'Neill L, Cervia J, Haller I, Giunta Y, Licholai T, Noel GJ. Energy balance, viral burden, insulin-like growth factor-1, interleukin- 6 and growth impairment in children infected with human immunodeficiency virus. AIDS 2000;14(6):683-90.

8. Vania L, Santos M, Nolasco M, Ancona F, Azevedo A. Evolution of nutritional status of infants infected with the human immunodeficiency virus. Sao Paulo Med J 2000;118(5):14853.

9. Villalobos D, Maury-Sintjago E, Ríos P, Fernández C, García D, Bravo A. Evaluación del estado nutricional en pacientes pediátricos institucionalizados con VIH/SIDA. Pediatr Asunción 2011;38(1):31-39.

10. Shet A, Mehta S, Rajagopalan N, Dinakar C, Ramesh E, Kurpad A. Anemia and growth failure among HIV-infected children in India: a retrospective analysis. BMC Pediatrics 2009;(9):37-45.

11. Duggan C. Micronutrients and Child Health: Studies in International Nutrition and HIV Infection. Nut Reviews 2001;59(11):358-69.

12. Vigano P, Berchielli J, Hjertquist T, Vidal B, García R, Pontes J. Nutritional status and lipid profile of HIV-positive children and adolescents using antiretroviral therapy. Clin Sci 2011;66(6):997-1002.

13. Delphine J, Lévine $M$, Ortega-Rodríguez E, Eaye A, Polak $\mathrm{M}$, Vilmer $\mathrm{E}$, Levy $\mathrm{C}$. Clinical and metabolic presentation of the lipodystrophic syndrome in HIV-infected children. AIDS 2000;14:2123-28.

14. Sánchez N. El síndrome de lipodistrofia y su relación con pacientes con VIH/SIDA y la terapia antirretroviral. Ciencias 2008;25(2):47-65.

15. Rabkin M, El-Sadr W, Abrams E. Manual Clínico Pediátrico. $1^{\circ}$ ed. Nueva York: Columbia; 2004.

16. Mehta M, Corkins R, Lyman B, et al. Defining Pediatric Malnutrition: A Paradigm Shift Toward Etiology-Related Definitions. JPEN J Parenter Enteral Nutr. Published online 25 March 2013.

17. Suverza A, Haua K. El ABCD de la evaluación del estado de nutrición. 1edición. México: Mc Graw Hill;2010.
18. World Health Organization. Concentraciones de hemoglobina para diagnosticar la anemia y evaluar su gravedad. WHO. 2011. (WHO/NMH/NHD/MNM/11.1)

19. Venkatesh K, Luriel M, Triche E, Bruyn G, Harwell J, McGarvey S, Gray G. Growth of infants born to HIV-infected women in South Africa according to maternal and infant characteristics. Trop Med Int Health. 2010;15(11):1364-74.

20. Fields-Garner C. Compendio de Conocimientos Sobre la Infección por el VIH y Temas Relacionados con Nutrición. WISHH 2006;1-47.

21. Arpadi S. Growth Failure in Children with HIV infection. JAIDS 2000;25(1):37-42.

22. Gibb DM, Duong T, Tookey PA, Sharland M, TudorWilliams $G$, Novelli V, et al. Decline in mortality, AIDS and hospital admissions in perinatally HIV-1 infected children inthe United Kingdom and Ireland. BMJ 2003;327(7422):1019.

23. Banjoko S, Oseni F, Togun R. Iron status in HIV-1 infection: implications in disease pathology. BMC Clinical Pathology 2012;12:26.

24. Bergersen BM, Sandvik L, Bruun JN, Tonstad S. Elevated Framingham risk score in HIV-positive patients on highly active antiretroviral therapy: results from a Norwegian study of 721 subjects. Eur J Clin Microbiol Infect Dis 2004;23:625-30.

25. Nekhai S, Kumari N, Dhawan S. Role of cellular iron and oxygen in the regulation of HIV-1infection. Future Virol 2013;8(3):301-311.

26. Hall V. Síndrome de Inmunodeficiencia Adquirida-Terapia Antirretroviral. Costa Rica: CIMED; 2003.

27. Gutiérrez $S$, De León $M$, Cuñetti L, Gutiérrez G, Giménez V, Quian J. Dislipemia y lipodistrofia en niños uruguayos VIH positivos en tratamiento antirretroviral. Rev Méd Urug 2006;22(3):197-202.

28. López P, Caicedo Y, Rubiano LC, Cortés CA, Valencia A, Ramírez $\mathrm{O}$, et al. Alteraciones metabólicas con terapia antirretroviral altamente efectiva en niños positivos para VIH, Cali, Colombia. Infect 2009;13(4):283-92.

29. Miller T, Somarriba G, Kinnamon D, Weinberg G, Friedman L, Scott G. The Effect of a Structured Exercise Program on Nutrition and Fitness Outcomes in Human Immunodeficiency Virus-Infected Children. AIDS Res Hum Retroviruses 2010;26(3):313-18.

30. Calza L, Manfredi R, Chiodo F. Hyperlipidaemia in patients with HIV-1 infection receiving highly active antiretroviral therapy: Epidemiology, pathogenesis, clinical course and management. Int J Antimicrob Agents 2003;22:89-99.

31. NOM-037-SSA2-2002, Para la prevención, tratamiento y control de las dislipidemias.

32. Rodríguez-Carranza SI, Aguilar-Salinas C. Anormalidades metabólicas en pacientes con infección por VIH. Rev invest clín 2004;56(2):193-208. 